\title{
Achromatic color categories
}

\author{
PAUL C. QUINN, B. R. WOOTEN, and EVETTE J. LUDMAN \\ Brown University, Providence, Rhode Island
}

\begin{abstract}
Sternheim and Boynton's (1966) continuous judgmental color-naming technique was used to assess the elemental nature of achromatic colors. Results from three subjects indicate that the names "black" and "white" are necessary and sufficient to describe achromatic test lights associated with the name "gray." On the basis of the criteria that establishes a color as elemental, black and white were confirmed as elemental, whereas gray was not. These findings support the assumption of various opponent processing models that there are only two elemental achromatic sensations. In addition, the results support Hering's (1920) view that black and white differ from the paired-chromatic opponent colors in that they are not mutually exclusive.
\end{abstract}

The number of colors that are considered to be elemental, in that they cannot be broken down into more basic perceptual components, has for some time been of considerable interest to psychologists (see Boring, 1942, for an excellent review of the issue). One of the main reasons for this interest is that most opponent color models postulate that there are six psychologically elemental colors: red, yellow, green, blue, black and white (see, e.g., Hurvich, 1981). According to this view, observers should be able to describe all colors by using percentages of the six terms, either singly or in combination. For example, a light of $600 \mathrm{~nm}$, although often conveniently labeled with the single term orange, should be describable with some combination of the terms red and yellow. Sternheim and Boynton (1966), using a continuous judgmental color-naming technique, have recently demonstrated that orange is, in fact, a composite hue, reducible to red and yellow. Similarly, Fuld, Wooten, and Whalen (1981) found that the colors violet and purple were not elemental; they could be described with percentages of the terms red and blue. Finally, Fuld, Werner, and Wooten (1983) obtained evidence suggesting that brown was an elemental color in that the terms black and yellow were not sufficient to describe it. This last finding has, however, been called into question by a recent report indicating that brown is not an elemental color, although it may differ, perhaps in terms of its susceptibility to linguistic response biases, from other nonelemental colors (Rosano, Wooten, \& Quinn, 1984). Taken together, then, the results of these recent studies have not found exception to the assumption of various opponent processing models that there are six elemental colors.

The purpose of the present study was to investigate whether gray was a visual quality that was different from black and white or whether gray was simply a perceptual mixture of black and white. This issue received considerable attention in the early part of the century (again

Reprint requests should be directed to B. R. Wooten, W. S. Hunter Laboratory of Psychology, Brown University, Providence, RI 02912. see, Boring, 1942). Dimmick (1920, 1925, 1929) and Rich $(1926,1928)$ argued that the black-gray-white series was actually two series-a black-gray series and a whitegray series. In addition, Müller (1896, as cited in Boring, 1942) posited a "brain gray" that is given by a constant cortical process and is "seen alone when the black and white processes are in exact equilibrium" (Boring, p. 133). In these three accounts, gray is considered to be an elemental color. On the other hand, several in vestigators have argued that gray is a perceptual mixture of black and white (Hering, 1920/1964; Ladd-Franklin, 1922; Michaels, 1925; Neifield, 1924). In these accounts, gray is not considered to be an elemental color. Hering, for example, argued that gray was reducible to black and white in the same way that orange is reducible to yellow and red. In Hering's view, then, black and white, although opponents in terms of their spatial antagonism, differ from the two chromatic opponent pairs (red-green, blueyellow), in that they are not mutually exclusive. According to Hering, black and white occur together in lights associated with the name gray. The results of the present study provide the first direct confirmation of Hering's views on gray; observers were able to completely describe lights associated with the name gray with varying amounts of black and white.

\section{METHOD}

At the outset of the method section, it may be helpful to review the continuous judgmental color naming technique that was first used by Sternheim and Boynton (1966) to establish whether a given color is elemental. The procedure involves presenting observers with a series of stimuli that vary in color. The observers are told that the total amount of color in each stimulus should be assigned a value of $100 \%$. The observers are given various color terms to describe each test light. They are told that, to describe them, they are to assign a percentage to each color term that represents the amount of that color sensation in each light. They are also told that these percentages do not have to sum to $100 \%$ if the names they are allowed to use are not sufficient to completely describe the light. Thus, if the available color terms are not sufficient to completely describe a series of test lights, a computed function is obtained that represents the amount that the sum of the percentages of the color 
terms assigned to each light falls short of 100 . A color is considered to be elemental if, for a session in which its name is prohibited from use, a computed function can be obtained that matches its response function when the color name is allowed. A color is considered to be nonelemental if, for a session in which its name is prohibited from use, a computed function cannot be computed that matches its response function when the color name is allowed. By this criterion, Sternheim and Boynton (1966) determined that yellow was an elemental hue, whereas orange was not. Observers showed that the terms red and green were not sufficient to describe a series of test lights ranging in wavelength from 530 to $620 \mathrm{~nm}$. A broad computed function that peaked at $580 \mathrm{~nm}$ was observed. This computed function closely matched the yellow response function obtained in a second session in which the terms red, yellow, and green were allowed. That the yellow response function corresponded with the computed function when the yellow response category was not allowed is, by Sternheim and Boynton's criterion, evidence that yellow is an elemental hue. Essentially no computed function was obtained in this second session, demonstrating that the terms red, yellow, and green were sufficient to completely describe the series of test lights. The term orange was added to the allowable response categories of red, yellow, and green in a later session. Although the term orange was used to describe lights previously described by the terms red and yellow, it was found to be redundant. The results of the second session demonstrated that the color terms red and yellow were sufficient to completely describe the lights associated with name orange. Because the orange response function displayed no correspondence with the computed function when orange was not allowed (essentially flat at zero), Sternheim and Boynton concluded that orange was not an elemental hue.

The present study applied the continuous judgmental color naming technique that was used to investigate the elemental nature of the hues in the yellow-orange-red chromatic series to the problem of determining the elemental nature of the colors in the black-graywhite achromatic series. The question of whether gray was an elemental color was examined by comparing the computed function obtained in a session in which only the response categories black and white were allowed, with the gray response function obtained in a session in which all three terms were allowed. Similarly, the elemental nature of white was determined by comparing the computed function obtained in a session in which only the term black was allowed, with the white response function obtained in sessions in which white was available singly or in combination with black. Finally, whether black was an elemental color was determined by comparing the computed function obtained in a session in which only white was allowed, with the black response function obtained in sessions in which black was available singly or in combination with white.

\section{Preliminary Experiment}

Subjects. Three experimentally naive subjects were used. All had normal color vision, as assessed by Ishihara pseudo-isochromatic plates.

Procedure. The three observers participated in a preliminary practice session using colored lights. This was designed to familiarize the subjects with the apparatus and the continuous judgmental color naming procedure, as well as to represent a modified replication of Sternheim and Boynton's (1966) investigation of the orange color naming category. The test stimuli were elliptical in shape and subtended $2.36^{\circ} \times 1.44^{\circ}$ of visual angle along the vertical and horizontal axes, respectively. They varied in wavelength from 570 to $650 \mathrm{~nm}$ (yellowish-green to yellowish-red) in steps of $10 \mathrm{~nm}$ and were equated for brightness on the basis of Wagner and Boynton's (1972) step-by-step matching function. The retinal illuminance of 570 -nm stimulus was set at $2.5 \log$ trolands.

The practice session began with $10 \mathrm{~min}$ of dark adaptation, after which an observer was shown six blocks of nine test stimuli. Within each block, the order of presentation of the nine test stimuli was randomized. The stimuli were presented for $2 \mathrm{sec}$, every $20 \mathrm{sec}$. The observers were allowed an additional presentation if they asked for it. The experimental room was dark except for a very low level of stray light reflected from optical elements. The observers were instructed to assign percentages to the color names yellow, green, and red in describing their perception of the central test field for the first three blocks of the nine test stimuli; in addition, the orange response category was allowed for the last three blocks. The observers were also instructed that the total of the percentages assigned to the different color names did not have to add up to $100 \%$ if the names they were allowed to use in the session were not sufficient to completely describe the light. In this practice session, results were obtained that were consistent with those of Sternheim and Boynton. The terms green, yellow, and red were sufficient to completely describe the series of test lights, whereas the term orange was redundant.

\section{Main Experiment}

Subjects. The three observers who participated in the preliminary experiment also participated in the main experiment.

Procedure. In order to generate the black-gray-white series, the central test field used in the preliminary experiment was surrounded with an annulus, the outside diameter of which subtended $5.58^{\circ}$ of visual angle. Both the central test field and the circular annulus presented achromatic light to the subject. There were 12 lights, 10 of which could be distinguished by the value of the surround illuminance, which ranged from 1.38 to $3.5 \mathrm{log}$ trolands. The retinal illuminance of the central test field was constant at $2.2 \mathrm{log}$ trolands. Two stimuli were added to allow subjects to view a broader perceptual continuum from white to black. Thus, for the extreme stimulus on the white end of the perceptual continuum, the light from the circular annulus was blocked and the central test field remained at $2.2 \mathrm{log}$ trolands. For the extreme stimulus on the black end of the perceptual continuum, light from the central test field was blocked, while the circular annulus was presented at $3.5 \mathrm{log}$ trolands. The 2 extreme stimuli were distinguished from the 10 intermediate stimuli in all of the figures by the placement of breaks in the appropriate places on the response functions and along the $\mathrm{x}$-axis.

On the day following the practice session, each observer began the main experiment by investigating the color name gray. The main experiment consisted of five sessions, each conducted on a separate day. In each session, responses were obtained for six blocks of 12 test lights. The test lights were presented in a random order within each block. As in the preliminary experiment, the test lights were presented for $2 \mathrm{sec}$, every $20 \mathrm{sec}$, in an otherwise dark room. The procedure for each of these sessions was identical, except that the names available to describe the central test field differed from day to day. The allowed color names varied in the following way: Session 1-black, white; Session 2-black; Session 3-white; Session 4-black, white, gray; Session 5-black, white. Observers were given the same instructions provided in the preliminary experiment. They were told to assign percentages to the available response categories that represented the amount of each of the color sensations perceived in a given test light. They were further told that these percentages did not have to sum to $100 \%$ if the names they were allowed to use in a session were not sufficient to completely describe the light. Finally, the observers were reminded to consider only the attributes of the central test field in making their judgments.

\section{Apparatus}

Three channels of a four-channel Maxwellian view optical system (described in detail by Knoblauch, 1981) were used. In the preliminary experiment, only one of the three channels was used. Light in this channel emanated from a xenon lamp source and provided a central test field that was rendered monochromatic by a grating monochromator. Two channels were used in the main experiment. Light for both channels was provided by a second xenon 
lamp source. The center-surround configuration was achieved by using a Lummer-Brodhan cube. Light in both channels was rendered white by interposing Wratten color-balancing filters until the center and surround were judged by the experimenters to be devoid of hue. The exit pupil of the optical system was $1.5 \mathrm{~mm}$ in diameter and was centered in the subject's pupil. The subject's head was held steady by an adjustable bitebar-and-headrest assembly. Retinal illuminance was determined by Westheimer's (1966) method.

\section{RESULTS}

At this point, it may be helpful to review the criteria for establishing a color as elemental. A color is considered to be elemental if, for a session in which its name is prohibited from use, a function can be computed by subtracting the sum of the percentages assigned to the available color names from 100 (for each test stimulus) that matches its response function when the color name is allowed. The question of whether gray is an elemental color can be determined by comparing the computed function from Session 1 , in which only the terms black and white were allowed, with the gray response function from Session 4, in which all three terms were allowed. In a similar manner, the assumed elemental nature of white (at least in opponent color theory) can be confirmed by comparing the computed function obtained in Session 2 (in which white was not allowed) with the white response functions obtained in Sessions 1 and 3. Finally, the assumed elemental nature of black can be confirmed by comparing the computed function obtained in Session 3 (black not allowed) with the black response functions obtained in Sessions 1 and 4.
Consider first the color-naming responses from Session 1 , in which the color names black and white were the only ones allowed. These responses are shown in the top panel of Figure 1, where percent color is plotted as a function of the retinal illuminance of the surround. At low values of surround retinal illumination, white was the predominant response. As the retinal illuminance of the surround was increased, however, the predominant white response gradually gave way to responses with approximately equal amounts of white and black, which themselves gave way to predominantly black responses. Figure 1 shows that black and white can be seen together in the same light. Figure 1 also shows that this series of lights can be completely described with the black and white response categories. The bottom panel of the figure shows that the computed function was essentially flat at zero percent across all values of retinal illuminance of the surround for the three subjects. Also plotted in the bottom panel of Figure 1 are the gray response functions obtained in Session 4, in which the categories black, white, and gray were allowed. That gray is not an elemental color is supported by the lack of correspondence between the computed function when the category was not allowed and the gray response function when it was allowed.

The top panel of Figure 2 displays the black responses obtained in Session 2, in which only the black response category was allowed. As was true in Session 1, the black response category, although little used when the retinal illuminance of the surround was low, comes to be relied on heavily as the retinal illuminance of the surround was increased. The computed function obtained in Session 2
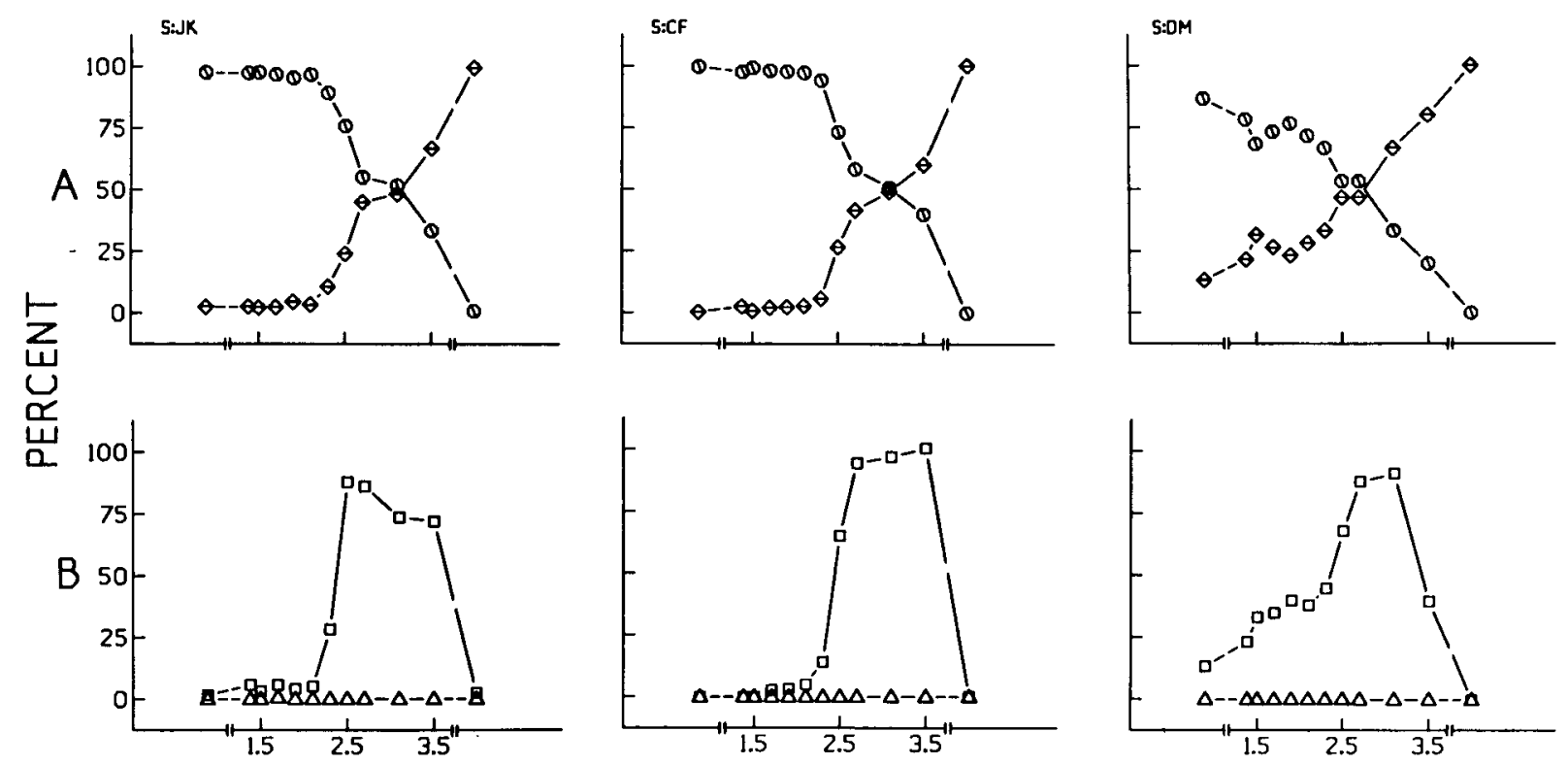

\section{SURROUND ILLUMINANCE, LOG TD}

Figure 1. (A) Color naming responses from Session 1 for the three subjects (allowed color names: black and white). White and black response functions are indicated by cross-hatched circles and diamonds, respectively. (B) Computed functions from Session 1 and gray response functions from Session 4 for these subjects. Computed and gray response functions are indicated by triangles and squares, respectively. 

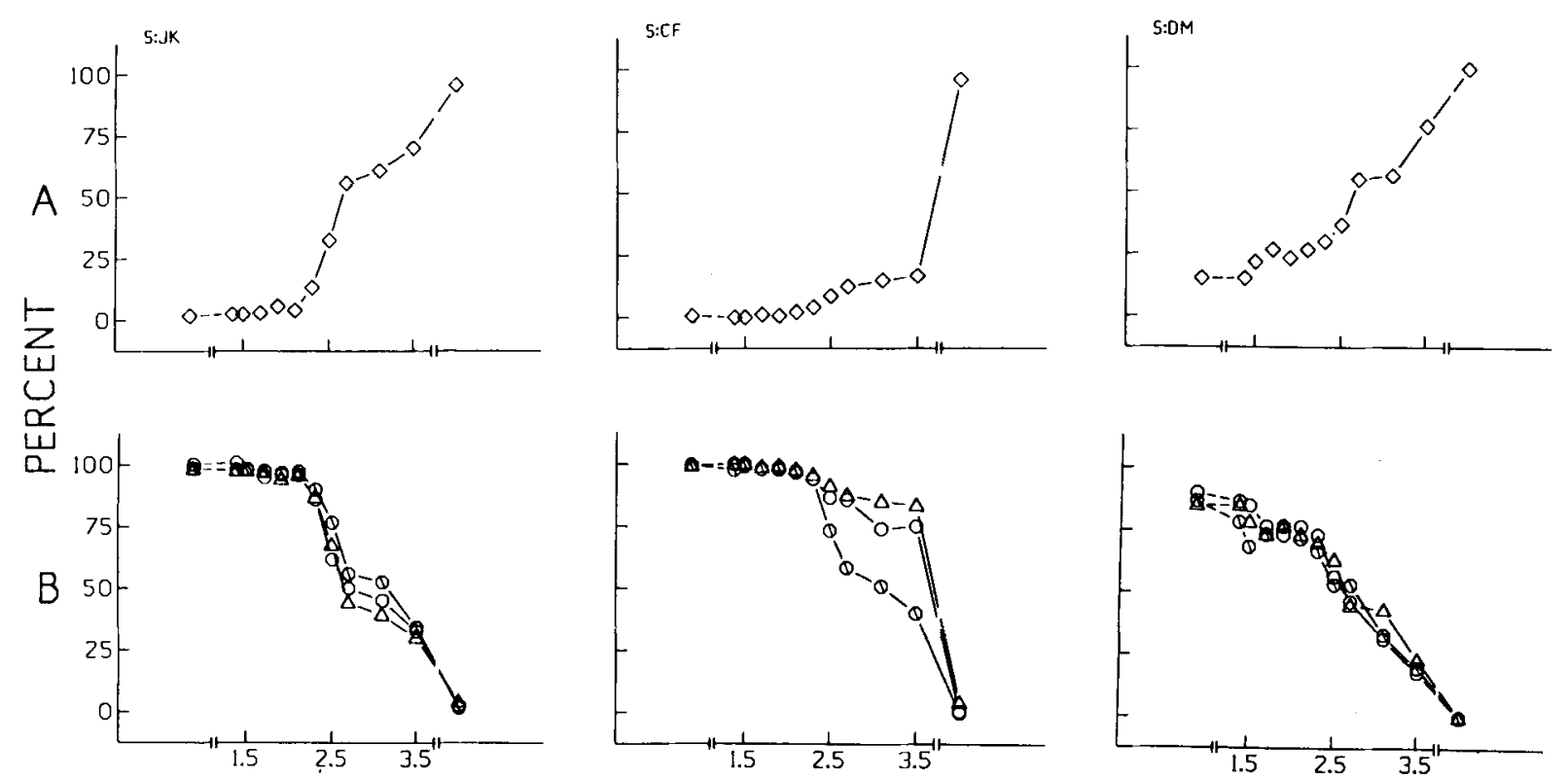

SURROUND ILLUMINANCE, LOG TD

Figure 2. (A) Color naming responses from Session 2 for the three subjects (allowed color name: black). Black response functions are indicated by diamonds. (B) Computed functions from Session 2 and white response functions from Sessions 1 and 3 for these subjects. Computed functions are represented by triangles; white response functions from Sessions 1 and 3 are represented by cross-hatched and unfilled circles, respectively.

can be seen in the bottom panel of Figure 2. The bottom panel also displays the white responses obtained in the first and third sessions. The data of Subjects J.K. and D.M. reveal an excellent correspondence between the computed function and the white response functions obtained in the two separate sessions. For Subject C.F., there also appears to be close agreement between the computed function and the white responses obtained in Session 3, although this correspondence breaks down slightly when the computed function is compared with the white responses obtained in Session 1. This breakdown in correspondence occurs only for intermediate values of the retinal illuminance of the surround; the two functions are in accord for higher and lower values. Overall, the data depicted in the bottom panel of Figure 2 provide evidence that white is an elemental color. The computed functions obtained from the sessions in which the white response category was not allowed match closely with the white response functions obtained in sessions in which the white response category was allowed.

The white response function obtained in Session 3, already displayed in the bottom panel of Figure 2, is replotted in Figure 3. As mentioned previously, lights that have surrounds with low retinal illuminance were judged to be predominantly white. It can be seen that this usage of the white response category decreases with increases in the retinal illuminance of the surround. In the bottom panel of Figure 3, the computed function obtained in the third session is plotted along with the black responses from Sessions 1 and 2. Here, again, one finds almost complete agreement between the computed function and the two black response functions for Subjects J.K. and D.M. For Subject C.F., this close match with the computed function is also true of the black responses obtained in Session 2 . The agreement between this subject's computed function and the black response function obtained in Session 1 is good, but breaks down somewhat at intermediate retinal illuminance values of the surround. When the patterns of responding of the three observers are considered together, however, black, like white, emerges as an elemental color. The black responses obtained when the black response category was allowed were almost identical to the computed function obtained when the black response category was not allowed.

Figure 4 shows the response functions obtained from Session 4. In this session, in addition to the response categories of white and black, subjects were allowed to use the response category gray to describe their percepts. Adding the response category gray leads to a reduction in the proportion of black and white responses over a wide range of retinal illuminance values of the surround. This can be seen most clearly for retinal illuminance values of the surround that range from about 2.5 to $3.1 \mathrm{log}$ trolands. In Session 1, these lights were described with varying amounts of white and black. Figure 4 shows that in Session 4, however, these lights were described as predominantly gray. How the use of the white and black response categories is affected by the use of the gray response category is further illustrated in Figure 5. This figure directly compares the white and black response functions obtained when gray was not allowed (Session 1) with those obtained when gray was allowed (Session 4). 

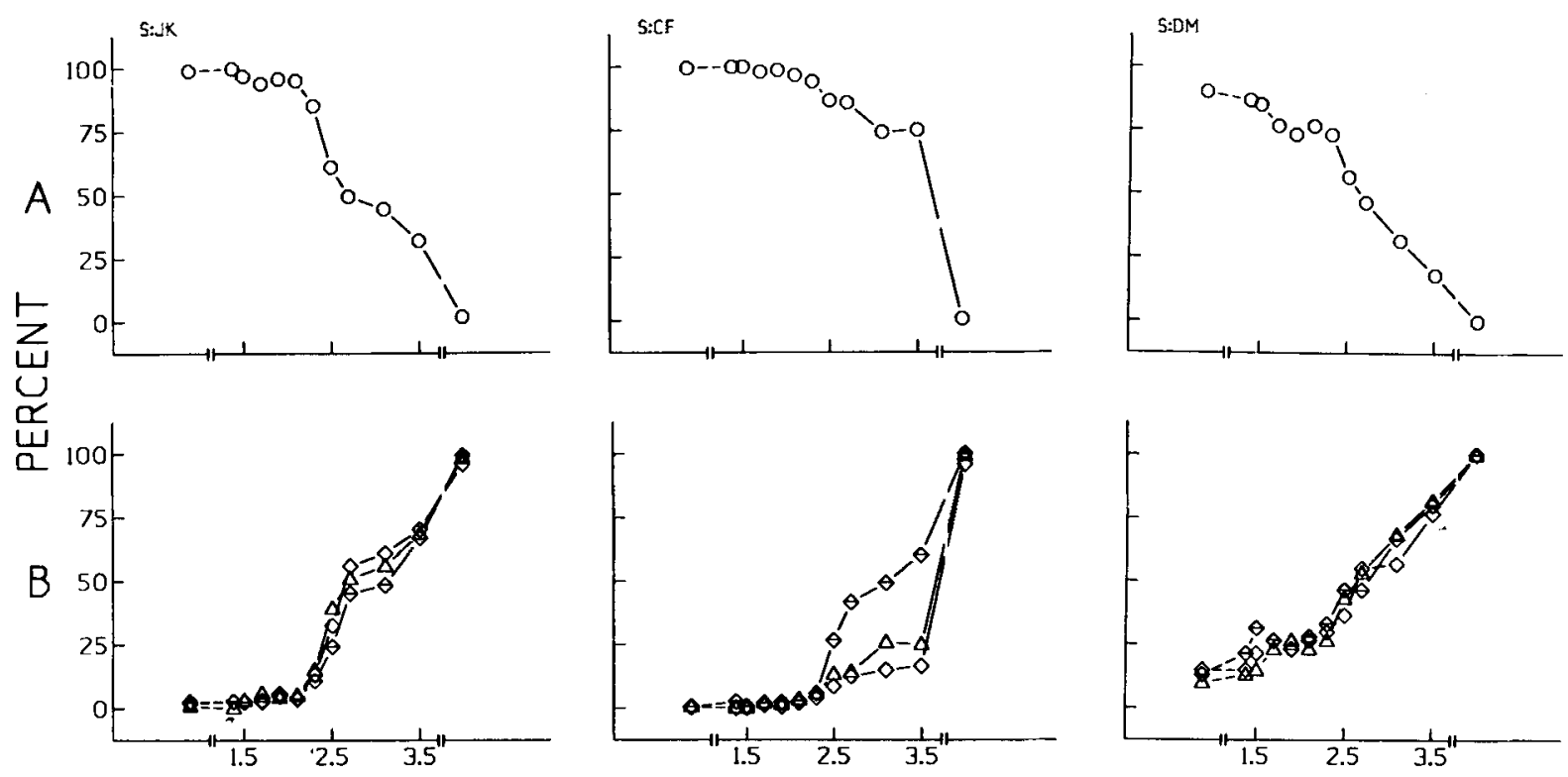

\section{SURROUND ILLUMINANCE, LOG TD}

Figure 3. (A) Color naming responses from Session 3 for the three subjects (allowed color name: white). White response functions are indicated by circles. (B) Computed functions from Session 3 and black response functions from Sessions 1 and 2 for these subjects. Computed functions are represented by triangles; black response functions from Sessions 1 and 2 are represented by cross-hatched and unrilled diamonds, respectively.

For all three subjects, it can be seen that with the use of the gray response category, the percentage of color allotted to the white response category first drops off at a lower value of surround retinal illuminance and decreases at a faster rate. The use of the gray response category also causes the percentage of color allotted to the black response category to begin to increase at a higher value of surround retinal illuminance and continue its increase at a faster rate. In other words, the use of the gray category reduces the proportion of both white and black responses. Finally, returning to Figure 4, it should be noted that, for each subject, the maximum gray response occurs at a retinal illuminance value of the surround where the black and white responses were at or close to their minimum value. No computed function was obtained from this session.

One might question whether, after experience using the gray response category, subjects might not be as willing as they were in Session 1 to completely describe the series of achromatic test lights with the terms black and white. This question was addressed by conducting a fifth and final session that was identical to the first session, in which only the black and white response categories were allowed. In Figure 6, the black and white response functions obtained in Session 1 have been replotted and can be compared with those obtained in Session 5 . The black
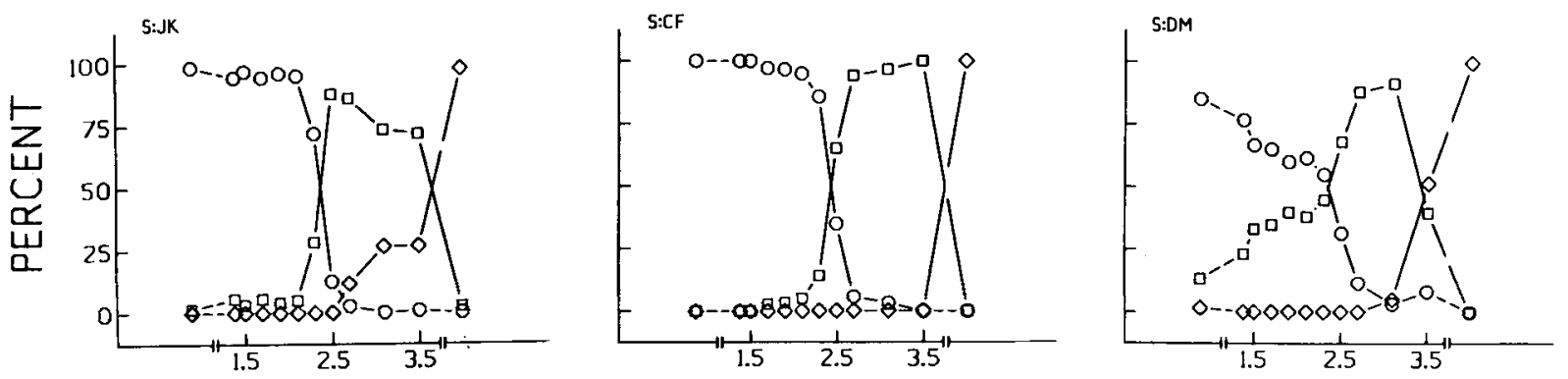

\section{SURROUND ILLUMINANCE, LOG TD}

Figure 4. Color naming responses from Session 4 for the three subjects (allowed color names: white, black, and gray). White, black, and gray response functions are indicated by the circles, diamonds, and squares, respectively. 

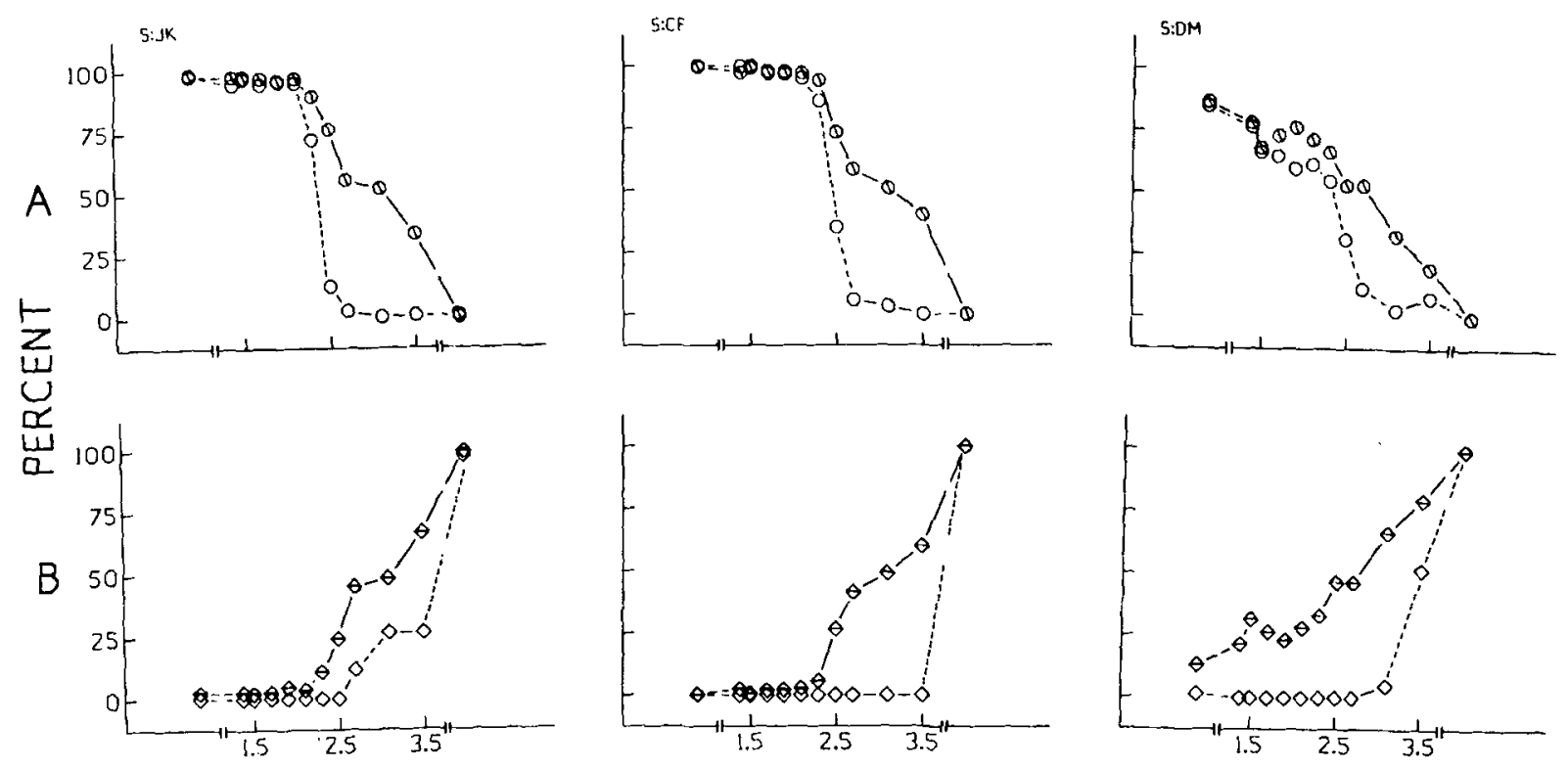

\section{SURROUND ILLUMINANCE, LOG TD}

Figure 5. Comparison of white response functions $(A)$ and black response functions $(B)$ from Session 1 , in which gray was not allowed (cross-hatched symbols, solid line), and Session 4, in which gray was allowed (unfilled symbols, dashed line), for the three subjects. White and black response functions are indicated by circles and diamonds, respectively.

and white response functions obtained in the fifth session were in excellent correspondence with those observed in Session 1. Once again, the black and white response categories were sufficient to completely describe these lights. As was the case for Session 1, essentially no computed function was obtained for any of the three subjects. The data obtained from Session 5 thus indicate that after experience using the color name gray, subjects were no less likely to use the black and white response categories. Such a result provides further evidence of the nonelemental status of gray.

\section{DISCUSSION}

The pattern of color naming responses obtained in the present study indicates that gray fails to meet the criteria that establishes a color as elemental. Although subjects made use of the gray response category when it was allowed, they found the terms black and white to be sufficient to completely describe the series of achromatic test stimuli when the gray response category was not allowed. The resulting lack of correspondence between the gray response function obtained in Session 4 and the essentially negligible computed functions obtained in Sessions 1 and 5 , when only the black and white response categories were allowed, provides strong evidence that gray is not an elemental color. Both black and white, on the other hand, were found to meet the criterion that establishes a color as being elemental. The computed functions obtained when the black and white response categories were not allowed closely match the respective response functions from sessions in which the terms were allowed. Overall,
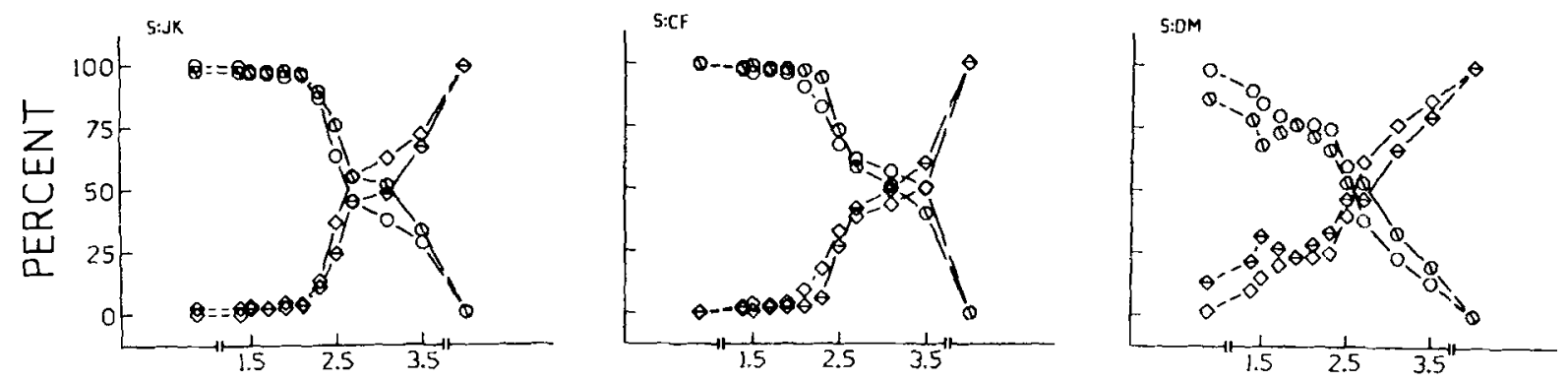

SURROUND ILLUMINANCE, LOG TD

Figure 6. Comparison of black and white response functions from the two sessions in which only these color names were allowed for the three subjects (Sessions 1 and 5). Responses from Sessions 1 and 5 are indicated by the cross-hatched and unfilled symbols, respectively. Black and white response functions are indicated by diamonds and circles, respectively. 
then, these findings uphold the assumption of various opponent processing models that all achromatic colors can be fully described with percentages of black and white (see, e.g., Hurvich, 1981).

The data obtained in the present study do not substantiate earlier arguments that gray is a visual quality that is different from black and white (Dimmick, 1920, 1925, 1929; Müller, 1896, as cited in Boring, 1942; Rich, 1926, 1928). It was mentioned previously that Dimmick and Rich argued that the series of qualities running from black through white is better viewed as two series rather than one. Both of them begin at gray; one series ranges from gray to white, the other from gray to black. This argument followed from Dimmick's (1925) phenomenal observation that:

whitelikeness does not appear at the first step away from black, nor until the series has been gone ralf through. When it does appear all blacklikeness has already disappeared. And the region between the disappearance of black and the appearance of white is occupied by a gray which is neither black nor white but only gray (p. 335).

Returning to Figure 4, one can see that Dimmick's observations are consistent with the response functions obtained when the response categories black, white, and gray were allowed. For all three observers, the black response function reached a minimum at the surround retinal illuminance value for which the white response function only begins to increase. At the surround retinal illuminance value for which the black and white response functions were at their respective minima, the responses of the three observers were predominantly gray. Where Dimmick appears to stand in contrast with the data presented here is not in his observations, but in the conclusions he drew from them. Although the gray response dominates when the black and white responses were minimal, gray is not a visual quality that is independent of black and white. Rather, as Figures 1 and 6 clearly demonstrate, gray is a perceptual mixture of black and white. Thus, although Dimmick correctly characterized how an achromatic series would be named with the response categories black, white, and gray, he appears to have erred in his conclusion that gray was an elemental color.

The present data confirm the view that gray is a composite perceptual mixture of black and white (Hering, 1920/1962; Ladd-Franklin, 1922; Michaels, 1925; Neifield, 1924). In addition, these data provide strong support for Hering's arguments that black and white, although opponents in terms of their spatial antagonism, differ in a fundamental way from the paired-opponent chromatic colors in terms of their mutual exclusivity. As Hering summarizes:

It seems highly striking from the start that between red and green, for example, there is not a series of intermediate chromatic colors as there is between red and yellow or between red and blue, that there are consequently no colors that appear simultaneously reddish and greenish, in the way that orange is simultaneously reddish and yellowish, or gray simultaneously whitish and blackish (p. 13).

Clearly, if black and white exist as a composite mixture in various shades of gray, they are not psychologically mutually exclusive, as are red and green and blue and yellow. The results of the present study thus provide the first direct confirmation of Hering's view on this issue.

\section{REFERENCES}

Boring, E. G. (1942). Sensation and perception in the history of experimental psychology. New York: Appleton-Century-Crofts.

Dimmick, F. L. (1920). A note on the series of blacks, greys and whites. American Journal of Psychology, 31, 301-302.

Dimmick, F. L. (1925). The series of blacks, grays and whites. Psychological Review, 32, 334-336.

Dimmick, F. L. (1929). A reinterpretation of the color-pyramid. Psychological Review, 36, 83-90.

Fuld, K., Werner, J. S., Wooten, B. R. (1983). The possible elemental nature of brown. Vision Research, 23, 631-637.

Fuld, K., Wooten, B. R., \& Whalen, J. J. (1981). Elemental hues of short-wave and extraspectral lights. Perception \& Psychophysics, 29, 317-322.

Hering, E. (1964). Outlines of a theory of light sense (L. M. Hurvich \& D. Jameson, Trans.). Cambridge, MA: Harvard University Press. (Work originally published 1920)

Hurvich, L. M. (1981). Color vision. Sunderland, MA: Sinauer Associates.

KNOBLAUCH, K. (1981). Analysis of opponent interactions in sex-linked dichromacy. Unpublished doctoral dissertation, Brown University.

LADD-FrankLIN, C. (1922). Tetrachromatic vision and the development theory of color. Science, $55,555-560$.

Michaels, G. M. (1925). Black: A non-light sensation. Psychological Review, 32, 248-250.

NeIFIELD, M. R. (1924). The Ladd-Franklin theory of the black sensation. Psychological Review, 31, 498-502.

RICH, G. J. (1926). Black and grey in visual theory. American Journal of Psychology, 37, 123-128.

Rich, G. J. (1928). An eclectic theory of vision. Psychological Review, 35, 311-318.

Rosano, J. L., Wooten, B. R., \& Quinn, P. C. (1984). Evidence that brown is not an elemental color. Paper presented to the Optical Society of America, San Diego.

STERNHEIM, C. E., BoynTON, R. M. (1966). Uniqueness of perceived hues investigated with a continuous judgmental technique. Journal of Experimental Psychology, 72, 770-776.

WAGNER, G., \& BoYNTON, R. M. (1972). Comparison of four methods of heterochromatic photometry. Journal of the Optical Society of America, 62, 1505-1515.

Westheimer, G. (1966). The maxwellian view. Vision Research, 6, 669-682. 